\title{
REMARK ON A PAPER OF Y. IKEBE
}

\section{IVAN SINGER}

In a recent paper, $Y$. Ikebe [3] has proved a theorem on characterization of best approximations by elements of linear subspaces in the space of all continuous real- or complex-valued functions on a compact space. In the present paper we want to observe that this result can be extended to arbitrary real or complex normed linear spaces. We shall use the notations of [5].

Theorem. Let $E$ be a normed linear space, $G$ a linear subspace of $E$ and $x \in E \backslash \bar{G}$. An element $g_{0} \in G$ is a best approximation of $x$ (i.e. $\left\|x-g_{0}\right\|$ $\left.=\inf _{g \in G}\|x-g\|\right)$ if and only if 0 belongs to the $\sigma\left(G^{*}, G\right)$-closure of the convex hull of the set

(1) $A=\left\{\left[f\left(x-g_{0}\right)\right]-\left.f\right|_{a}\left|f \in \varepsilon\left(S_{E^{*}}\right),\right| f\left(x-g_{0}\right) \mid=\left\|x-g_{0}\right\|\right\}$,

where $\mathcal{E}\left(S_{E^{*}}\right)$ denotes the set of all extreme points of the unit cell $S_{E^{*}}$ $=\left\{f \in E^{*} \mid\|f\| \leqq 1\right\},\left.f\right|_{a}$ denotes the restriction of $f$ to the subspace $G$ and where [ ]- stands for complex conjugate.

Proof. Let us denote by $\Omega(A)$ the $\sigma\left(G^{*}, G\right)$-closure of the convex hull of $A$.

Necessity. Assume that $0 \notin \Omega(A)$. Then, as observed also in [3], there exists an element $g_{1} \in G$ such that

$$
\sup _{\phi \in \Omega(A)} \operatorname{Re} \phi\left(g_{1}\right)<0 .
$$

Now, if $g_{0}$ would be a best approximation of $x$, then, by $[\mathbf{5}, \mathrm{pp} .57-$ 58 , Corollary 1.9], for every $g \in G$ there would exist an $f^{\circ} \in \mathcal{E}\left(S_{E^{*}}\right)$ such that

$$
\begin{aligned}
& \left|f^{o}\left(x-g_{0}\right)\right|=\left\|x-g_{0}\right\|, \\
& \operatorname{Re}\left(\left[f^{o}\left(x-g_{0}\right)\right]-f^{o}(g)\right) \geqq 0,
\end{aligned}
$$

whence, for $\phi^{0}=\left[f^{\circ}\left(x-g_{0}\right)\right]-\left.f^{\circ}\right|_{G}$ one would obtain

$$
\phi^{\circ} \in A, \quad \operatorname{Re} \phi^{o}(g) \geqq 0,
$$

which contradicts (2).

Sufficiency can be proved similarly to [3]: assuming that $0 \in \Omega(A)$ and using the $\sigma\left(E^{*}, E\right)$-compactness of $S_{E^{*}}$, one easily obtains an $f \in E^{*}$ such that

Received by the editors December 10, 1967. 


$$
\begin{gathered}
\|f\|=1, \quad f(g)=0 \quad(g \in G), \\
f\left(x-g_{0}\right)=\left\|x-g_{0}\right\|,
\end{gathered}
$$

whence [4] $g_{0}$ is a best approximation of $x$. This completes the proof of the theorem.

In the particular case when $E=C(Q)$, the space of all continuous real- or complex-valued functions on a compact space $Q$, we have $f \in \mathcal{E}\left(S_{E^{*}}\right)$ if and only if $f$ is of the form $\alpha_{0} \epsilon_{g_{0}}$, i.e.

$$
f(y)=\alpha_{0} y\left(q_{0}\right) \quad(y \in C(Q)),
$$

where $\alpha_{0}$ is a scalar with $\left|\alpha_{0}\right|=1$ and $q_{0} \in Q$ (see e.g. $[2$, p. 441, Lemma 6], or, for another proof, [5, p. 73]). Therefore

$$
\begin{aligned}
& A=\left\{\left[\alpha\left(x(q)-g_{0}(q)\right)\right]-\left.\alpha \epsilon_{q}\right|_{G}|| \alpha \mid=1, q \in Q,\right. \\
&\left.\left|x(q)-g_{0}(q)\right|=\left\|x-g_{0}\right\|\right\} \\
&=\left\{\left[x(q)-g_{0}(q)\right]-\left.\epsilon_{q}\right|_{a}|q \in Q,| x(q)-g_{0}(q) \mid=\left\|x-g_{0}\right\|\right\},
\end{aligned}
$$

and thus the above theorem yields the result of Y. Ikebe [3].

In the particular case when $\operatorname{dim} G=n<\infty$, the corollary given in [3] with reference to E. W. Cheney [1] can be also extended to arbitrary normed linear spaces as follows:

CoROllary. Let $E$ be a normed linear space, $G$ a linear subspace of $E$ with $\operatorname{dim} G=n<\infty$, and $x \in E \backslash G$. An element $g_{0} \in G$ is a best approximation of $x$ if and only if 0 belongs to the convex hull of the set (in the n-space)

$$
\begin{array}{r}
B=\left\{\left[f\left(x-g_{0}\right)\right]-f\left(x_{1}\right), \cdots,\left[f\left(x-g_{0}\right)\right]-f\left(x_{n}\right) \mid f \in \mathcal{E}\left(S_{B^{*}}\right),\right. \\
\left.\left|f\left(x-g_{0}\right)\right|=\left\|x-g_{0}\right\|\right\},
\end{array}
$$

where $x_{1}, \cdots, x_{n} \in G$ is some basis of $G$.

This corollary can be deduced from the above theorem as in the corresponding particular case in [3], observing that if $\operatorname{dim} G<\infty$, then the convex hull of the set $A$ defined by (1) is $\sigma\left(G^{*}, G\right)$-closed and using the isomorphic mapping $\phi \rightarrow\left(\phi\left(x_{1}\right), \cdots, \phi\left(x_{n}\right)\right)$ of $G^{*}$ onto the $n$-space.

\section{REFERENCES}

1. E. W. Cheney, Introduction to approximation theory, McGraw-Hill, New York, 1966.

2. N. Dunford and J. Schwartz, Linear operators. Part I, Interscience, New York, 1958. 
3. Y. Ikebe, A characterization of best Tchebycheff approximations in function spaces, Proc. Japan Acad. (1968).

4. I. Singer, Caractérisation des éléments de meilleure approximation dans un espace de Banach quelconque, Acta Sci. Math. Szeged 17 (1956), 181-189.

5. - Best approximation in normed linear spaces by elements of linear subspaces, Ed. Acad. Repub. Socialiste Romania, 1967. (Romanian)

Institute of Mathematics of The Romanian Academy of Sciences, BUCHAREST 